\title{
Matrix metalloproteinase-2 regulates MDA-MB-231 breast cancer cell invasion induced by active mammalian diaphanous-related formin 1
}

\author{
DAEHWAN KIM and SANGMYUNG RHEE
}

Department of Life Science, Chung-Ang University, Seoul 06974, Republic of Korea

Received April 24, 2015; Accepted February 23, 2016

DOI: $10.3892 / \mathrm{mmr} .2016 .5282$

\begin{abstract}
Mammalian diaphanous-related formin 1 (mDia1) was initially identified as a Rho GTPase effector involved in the progression of various diseases, including types of cancer. However, the precise underlying molecular mechanism of mDia1-mediated cancer cell invasion remains to be elucidated. In the present study, mDial expression was demonstrated to be upregulated in tissues from a number of cancer types, including kidney, prostate, and breast cancer using immunohistochemical analysis. Forced expression of a constitutively active (CA) form of mDial induces invasion, as measured by Transwell invasion assay, of MDA-MB-231 cells, which is a highly invasive breast cancer cell line, and this effect was markedly impaired by matrix metalloproteinase (MMP)-2 silencing. Furthermore, the present study demonstrated that overexpression of the CA form of mDial leads to the induction of invasive ability in MCF-7 cells, which is a non-invasive breast cancer cell line, as a result of increased MMP-2 activity. Thus, the results of the current study suggest that mDial is an important regulator of breast cancer cell invasion and that this effect may be mediated by MMP-2 activity.
\end{abstract}

\section{Introduction}

Invasion and metastasis are key mechanisms for the spread of cancer to new tissues or organs, and are major causes of cancer-associated mortality, including in breast cancer (1). Reorganization of cytoskeletal proteins, including actin and microtubule proteins, is important for the invasion and migration of cancer cells $(2,3)$. Formin proteins are essential for the

Correspondence to: Professor Sangmyung Rhee, Department of Life Science, Chung-Ang University, 84 Heukseok-Ro, Donjak-Gu, Seoul 06974, Republic of Korea

E-mail: sangmyung.rhee@cau.ac.kr

Abbreviations: mDia1, mammalian diaphanous-related formin 1; MMP-2, matrix metalloproteinase-2

Key words: formin, mammalian diaphanous-related formin 1, breast cancer cells, matrix metalloproteinase-2, invasion regulation of cytoskeleton remodeling $(4,5)$ and mammalian diaphanous-related formin 1 (mDia1), a member of the formin family, is a regulator of actin polymerization and microtubule stabilization via its formin homology 2 domain (6,7). mDial affects actin and microtubule dynamics; thus, it has various functions, including the regulation of adhesion, polarity, morphogenesis, intracellular trafficking of vesicles, and invasion (8-11). Although the roles of mDial in cytoskeleton modification have been well established, few reports have demonstrated its role in disease processes, including cancer progression.

Cancer cell invasion across tissue boundaries and metastasis via the circulatory system requires proteolytic activity to degrade extracellular matrix components. The matrix metalloproteinase (MMP) family is important in the spread of cancer to other tissues, MMPs degrade the extracellular matrix, including collagen, which is the first barrier preventing cancer invasion (12). Due to their effects on the invasion and metastasis of cancer cells, the functions of matrix metalloproteinases have been well studied (13-15). Among the MMPs, the expression levels of MMP-2 and MMP-9 are associated with unfavorable outcomes in various types of cancer cells, including breast cancer cells $(16,17)$. Despite their importance in cancer invasion, the association between mDia1 and MMPs has not been fully elucidated.

The present study aimed to identify underlying mechanisms of mDial to regulate cancer cell invasion. Immunohistochemical results from the cancer tissue array demonstrated that mDial expression levels are increased in a number of samples originating from kidney, prostate, and breast cancer. Experiments using forced expression of the constitutively active (CA) form of mDial (mDia1-CA) and MMP silencing in invasive and non-invasive breast cancer cell lines demonstrated the mDia1-MMP2 signaling axis is likely to be an important regulator of breast cancer invasion.

\section{Materials and methods}

Reagents. TransFectin Lipid reagent was obtained from Bio-Rad Laboratories, Inc. (Hercules, CA, USA). Oligofectamine Transfection reagent was obtained from Invitrogen (Thermo Fisher Scientific, Inc., Waltham, MA, USA). Fetal bovine serum (FBS) was purchased from Biowest LLC (Riverside, MO, USA). Polyclonal goat anti-mDial (dilution, 
1:1,000; cat. no. sc-10888) and monoclonal mouse anti-green fluorescent protein (GFP; dilution, 1:1,000; cat. no. sc-9996) antibodies were obtained from Santa Cruz Biotechnology, Inc. (Dallas, TX, USA); monoclonal mouse anti- $\alpha$-tubulin antibody (dilution, 1:1,000; cat. no. T9026) was obtained from Sigma-Aldrich (St. Louis, MO, USA) and polyclonal rabbit anti-detyrosinated $\alpha$-tubulin (dilution, 1:1,000; cat. no. AB3201) was purchased from EMD Millipore (Billerica, MA, USA). Rat tail type I collagen and Matrigel were purchased from BD Biosciences (Franklin Lakes, NJ, USA). Formalin-fixed paraffin-embedded tissue microarray slides containing 30 tissue samples from healthy individuals or samples of normal tissue adjacent to cancer and 29 cancer tissue samples were obtained from SuperBioChips Laboratories (Seoul, Korea).

Cell culture. MDA-MB-231 and MCF-7 breast cancer cell lines were purchased from Korean Cell Line Bank (Seoul, Korea). All cell lines were cultured in RPMI 1640 (Invitrogen; Thermo Fisher Scientific, Inc.) supplemented with 10\% FBS, $100 \mathrm{U} / \mathrm{ml}$ penicillin, and $100 \mu \mathrm{g} / \mathrm{ml}$ streptomycin (Welgene, Inc., Daegu, Korea). Cell culture and incubations were performed at $37^{\circ} \mathrm{C}$ in a $5 \% \mathrm{CO}_{2}$ incubator.

Plasmids and transfection. The GFP-tagged CA form of mDial was provided by Dr. Sohail Ahmed (Institute of Medical Biology, Singapore). The sequences of mDia1-CA construct were amplified by PCR and inserted into XhoI/BamHI (New England BioLabs, Inc., Medford, NJ, USA)-digested pEGFP-N1 (Clontech Laboratories, Inc., Mountainview, CA, USA) to produce mDia1-CA (GFP). An EGFP empty vector served as a mock control. All clone sequences were verified by DNA sequencing (conducted by Cosmo Genetech Co., Ltd., Seoul, Korea). Transient transfection was performed using TransFectin Lipid reagent or a Neon Electroporation system (Thermo Fisher Scientific, Inc.) according to the manufacturer's protocols.

siRNA transfection. MMP-2 small interfering RNA (siRNA; sequence, 5'-AAUACAUCGAGACCAUGCGG-3'), MMP-9 siRNA (sequence, 5'-AACAUCACCUAUUGGAUCCAA-3'), and non-targeting control (mock) siRNA (sequence, 5'-AUU GUAUGCGAUCGCAGAC-3') were purchased from Genolution, Inc. (Seoul, Korea). Cells were transfected with $20 \mathrm{nM}$ siRNA (unless otherwise stated) using Oligofectamine reagent according to the manufacturer's protocols.

Reverse transcription-polymerase chain reaction $(P C R)$. Total RNA was isolated from cells using RNAiso Plus (Takara Bio, Inc., Otsu, Japan) and cDNA was synthesized using Ex-Taq DNA Polymerase (Takara Bio, Inc.) according to the manufacturer's protocols. The conditions were 30 cycles of $94^{\circ} \mathrm{C}$ for $30 \mathrm{sec}, 55^{\circ} \mathrm{C}$ for $30 \mathrm{sec}$ and $72^{\circ} \mathrm{C}$ for $30 \mathrm{sec}$. PCR amplification was conducted using Ex-Taq DNA Polymerase (Takara Bio, Inc.) and SYBR Premix Ex-Taq II (Takara Bio, Inc.) according to the manufacturer's protocol. The reaction was conducted in a CFX Connect ${ }^{\mathrm{TM}}$ Real-Time PCR Detection system (Bio-Rad Laboratories, Inc.), with conditions as follows: 40 cycles of $94^{\circ} \mathrm{C}$ for $10 \mathrm{sec}, 55^{\circ} \mathrm{C}$ for $10 \mathrm{sec}$ and $72^{\circ} \mathrm{C}$ for $30 \mathrm{sec}$. Oligonucleotide sequences of primers used in the present study were as follows: Sense, 5'-GGCCCTGTCACTCCTGAGAT-3' and antisense, 5'-GGC ATCCAGGTTATCGGGGA-3' for MMP-2; sense, 5'-CAT AAGGACGACGTGAATG-3' and antisense, 5'-GAAGAA AAGCTTCTTGGAGA-3' for MMP-9; sense, 5'-GAG TCAACGGATTTGGTCGT-3' and antisense, 5'-TGTGGT CATGAGTCCTTCCA-3' for GAPDH. GAPDH served as an internal control and total RNA was used as a negative control. The results of the PCR were run on agarose gels and band intensity was quantified using Quantity One software (version 4.6.2; Bio-Rad Laboratories, Inc.).

Immunohistochemistry. Formalin-fixed, paraffin-embedded tissue microarray slides from cases of kidney, breast, prostate, endometrial and thyroid cancer were de-paraffinized by incubation in a dry oven for $1 \mathrm{~h}$, rehydrated with serially diluted ethanol, and blocked with normal goat serum (Vector Laboratories, Inc., Burlingame, CA, USA) at room temperature for $30 \mathrm{~min}$. Antigen retrieval in tissue samples was performed by heating in a microwave on high for $5 \mathrm{~min}$, medium for $5 \mathrm{~min}$ and low for $5 \mathrm{~min}$, followed by incubation with the anti-mDial antibody. Subsequently, the samples were incubated with a biotinylated polyclonal horse anti-goat (dilution, 1:200; cat. no. PK-6105), polyclonal goat anti-mouse (dilution, 1:5,000; Jackson ImmunoResearch Laboratories, Inc., West Grove, PA, USA; cat. no. 115-035-006) and polyclonal goat anti-rabbit (dilution, 1:5,000; Jackson ImmunoResearch Laboratories, Inc., West Grove, PA, USA; cat. no. 111-035-003) secondary antibodies, and streptavidin-horseradish peroxidase (Zymed; Thermo Fisher Scientific, Inc.). 3,3'-Diaminobenzidine (Vector Laboratories, Inc.) was used as a chromogen and eosin (Sigma-Aldrich) was used for counterstaining. Tissue sections were visualized by microscopy (Eclipse 80i; Nikon Corporation, Tokyo, Japan), and images were captured using a digital camera (Digital Sight DS-Qi1Mc; Nikon Corporation) and NIS Elements Image Analysis software (Nikon Corporation). The level of mDial expression was assessed by measuring staining intensity using Quantity One software (version 4.6.2).

Transwell cell invasion assay. Transwell inserts (Corning Incorporated, Corning, NY, USA) with an $8-\mu \mathrm{m}$ pore size were coated with $20 \mu \mathrm{g}$ type I collagen at $37^{\circ} \mathrm{C}$ overnight. Cells were placed in the upper chamber and RPMI 1640 supplemented with $10 \%$ FBS was added to the lower chamber as a chemoattractant and incubated for $24 \mathrm{~h}$. Mock siRNA of EGFP empty vector were used as controls. To demonstrate the function of MMPs, $5 \mu \mathrm{M}$ GM6001 (EMD Millipore) was added to the upper and lower chambers to prevent MMP-dependent invasion. Non-migrating or non-invading cells were removed with a cotton swab and cells were fixed with $100 \%$ methanol (Sigma-Aldrich for $10 \mathrm{~min}$ at room temperature, stained with $0.1 \%$ crystal violet (Sigma-Aldrich) and counted by light microscopy (IMT-2; Olympus Corporation, Tokyo, Japan). A quantitative analysis of counted cells per field and representative images are presented.

Gelatin zymography assay. Cells were seeded in a 35-mm dish and incubated in RPMI 1640 supplemented with 10\% FBS at $37^{\circ} \mathrm{C}$ for $24 \mathrm{~h}$. The cells were washed, and the medium was 


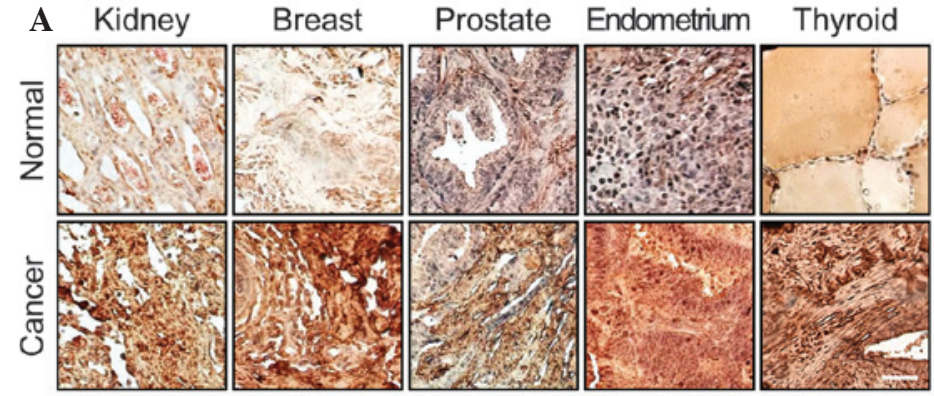

B

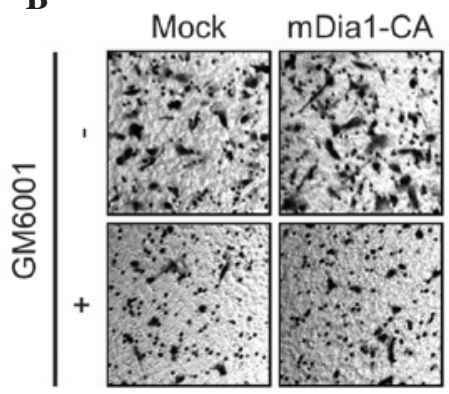

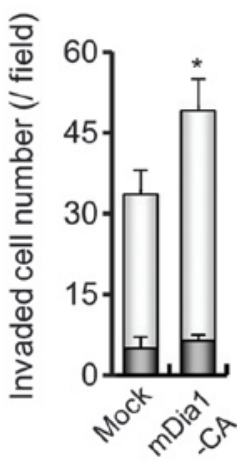

C
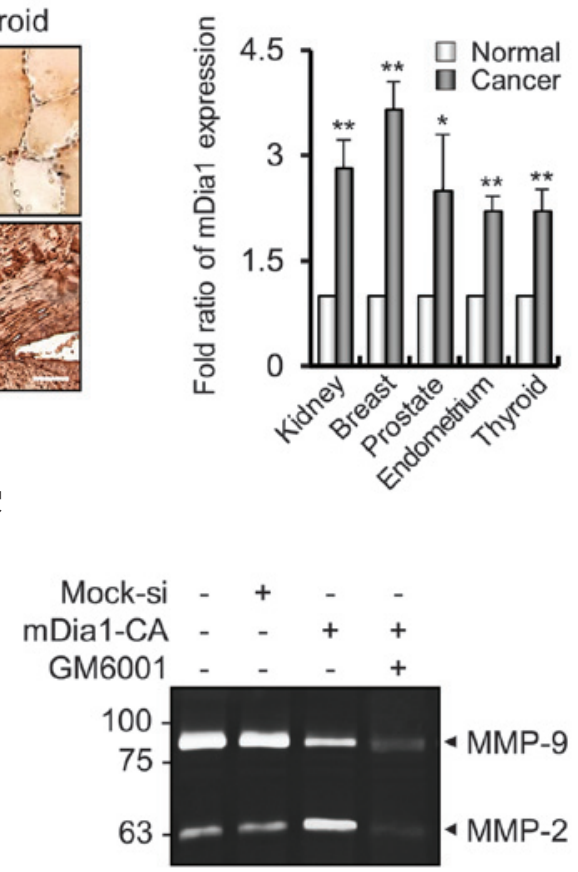

Figure 1. mDial influences breast cancer cell invasion. (A) Formalin-fixed tissue microarray slides were used in immunohistochemistry experiments. mDial was overexpressed in kidney, breast, prostate, endometrium, and thyroid cancer tissue samples, relative to the expression levels in normal tissues. "P $<0.05$, ${ }^{* *} \mathrm{P}<0.01$ vs. normal tissue samples. (B) Transwell invasion assays were performed with MDA-MB-231 cells overexpressing mDia1-CA. Invasion was considerably increased by forced expression of mDia1-CA. Filled bars indicate invaded cells in the presence of $5 \mu \mathrm{M}$ GM6001. "P<0.05 vs. the mock group. (C) Conditioned media from MDA-MB-231 cells were normalized based on cell number prior to performing gelatin zymography. Activity of MMP-2 was increased by overexpression of mDia1-CA, whereas MMP-9 activity was decreased. Activity of MMP-2 and MMP-9 were markedly decreased following treatment with $5 \mu \mathrm{M}$ GM6001. mDia1, mammalian diaphanous-related formin 1; CA, constitutively active; MMP, matrix metalloproteinase.
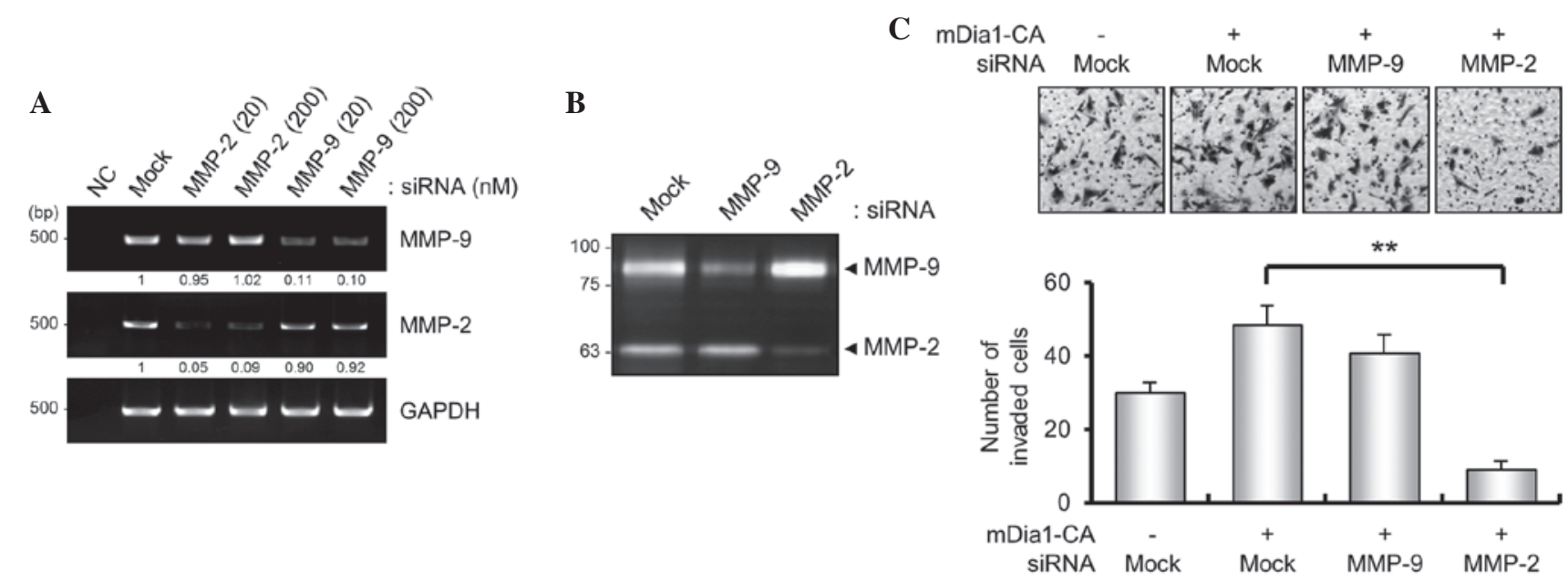

Figure 2. Breast cancer cell invasion mediated by mDia1 is dependent on MMP-2. (A and B) MDA-MB-231 cells were transfected with MMP-2- and MMP-9-specific siRNAs, and reverse transcription-polymerase chain reaction, zymography, and invasion assays were performed. Each siRNA strongly inhibited the expression and activity of its own locus, and did not change the (A) expression or (B) activity of the other MMP. (C) MDA-MB-231 cells overexpressing mDia1-CA were transfected with MMP-2 and MMP-9 siRNA, and then Transwell assays were performed. Knockdown of MMP-2 led to a marked reduction of MDA-MB-231 cell invasion promoted by mDia1-CA, whereas MMP-9 knockdown did not show considerable differences. ${ }^{* *} \mathrm{P}<0.01$. siRNA, small interfering RNA; MMP, matrix metalloproteinase; mDia1, mammalian diaphanous-related formin 1; NC, negative control; CA, constitutively active.

changed to serum-free RPMI 1640. Following incubation for 24 or $48 \mathrm{~h}$, the conditioned medium was centrifuged at $2,000 \mathrm{x}$ g for $5 \mathrm{~min}$ to remove cellular debris and was normalized based on the cell number prior to electrophoresis in $10 \%$ polyacrylamide gels with $1 \mathrm{mg} / \mathrm{ml}$ gelatin (Sigma-Aldrich).
Following $10 \%$ sodium dodecyl sulfate polyacrylamide gel electrophoresis for $90 \mathrm{~min}$ at $30 \mathrm{~mA}$, the gels were washed 4 times for $1 \mathrm{~h}$ with washing buffer $[10 \mathrm{mM}$ Tris- $\mathrm{HCl}(\mathrm{pH} 8.0)$ and $2.5 \%$ Triton $\mathrm{X}-100]$, rinsed with distilled water twice for $10 \mathrm{~min}$, and then incubated for $20 \mathrm{~h}$ in the developing 


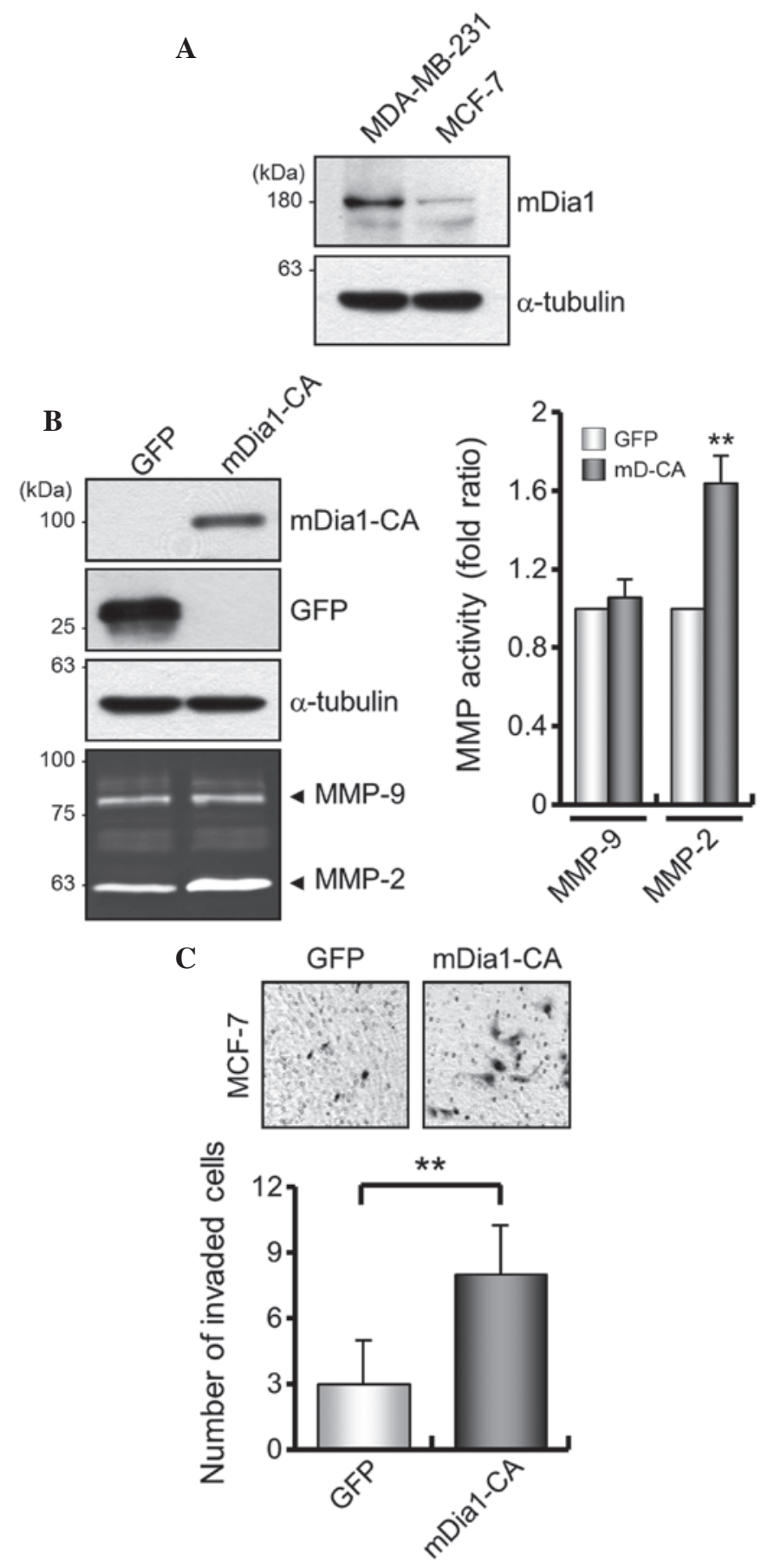

Figure 3. Active mDial induces invasion of non-invasive breast cancer cells by modulating MMP-2 activity. (A) Western blotting results for mDia1 expression in MDA-MB-231 and MCF-7 cells. (B and C) MCF-7 cells transfected with vector alone or mDia1-CA were subjected to zymography and invasion assays. Overexpression of mDia1-CA resulted in increased activity of MMP-2, but not MMP-9. Transwell assay indicated that invasion of MCF-7 cells expressing mDia1-CA is promoted markedly more than invasion of MCF-7 cells expressing GFP alone. ${ }^{* *} \mathrm{P}<0.01$ vs. the GFP group. MMP, matrix matalloproteinase; mDial, mammalian diaphanous-related formin 1; CA, constitutively active; GFP, green fluorescent protein.

buffer [50 mM Tris- $\mathrm{HCl}(\mathrm{pH} 7.5), 5 \mathrm{mM} \mathrm{CaCl}_{2}$, and $0.02 \%$ $\mathrm{NaN}_{3}$ ]. Gels were stained with $0.5 \%$ Coomassie blue R-250 (Sigma-Aldrich) for $10 \mathrm{~min}$ and destained with a solution of $10 \%$ methanol and $10 \%$ acetic acid until the gelatinolytic bands were visualized.
Statistical analysis. The differences between controls and treatment groups were analyzed by Student's t-test using Microsoft Excel software. The data were expressed as the mean \pm standard deviation of three independent experiments. $\mathrm{P}<0.05$ was considered to indicate a statistically significant difference.

\section{Results and Discussion}

mDial controls invasion of breast cancer cells via MMPs. To compare the mDial protein expression levels between normal and cancer tissue samples, immunohistochemical analysis was performed using a tissue microarray chip that contained 30 healthy individuals or normal tissue adjacent to the cancer and 29 cancer tissue samples. Among the tested samples, mDia1 expression was upregulated in a number of cancers, and particularly breast cancer tissue samples (Fig. 1A). To examine the effect of mDia1-CA overexpression on breast cancer cell invasion, highly invasive MDA-MB-231 cells were transfected with the mDia1-CA plasmid and the invasive ability was examined. As presented in Fig. 1B, forced expression of mDial-CA resulted in higher breast cancer cell invasion than that of mock-transfected cells. As mDial knockdown results in decreased breast cancer cell invasion (data not shown) (18), it is likely that mDial is directly involved in breast cancer cell invasion.

It is well known that MMPs are required to induce invasion of various cancers $(19,20)$. Thus, invasion assays were performed following treatment with a general MMP inhibitor, GM6001, using MDA-MB-231 cells transfected with either mock or mDia1-CA plasmids. The invasion of MDA-MB-231 cells induced by overexpression of mDia1-CA was markedly reduced in mock- and mDial-CA-transfected cells following GM6001 treatment (Fig. 1B). Furthermore, gelatin zymography using conditioned media prepared from the mock- and mDia1-CA-transfected cells demonstrated that MDA-MB-231 cells secreted MMP-2 and MMP-9, and that MMP-2 activity was markedly increased by mDia1-CA overexpression (Fig. 1C), indicating that the invasion of MDA-MB-231 cells regulated by mDial may be dependent on MMP activity.

MMP-2 is involved in MDA-MB-231 cell invasion via mDial. To investigate whether MMP activity is specifically required for mDia1-mediated cancer invasion, siRNAs for MMP-2 or MMP-9 were transfected into MDA-MB-231 cells overexpressing mDia1-CA and cell invasion was analyzed. Each siRNA decreased the transcript levels of MMP-2 and MMP-9 by $>90 \%$ (Fig. 2A). Furthermore, the results of gelatin zymography performed using the conditioned media obtained from the siRNA-transfected cells demonstrated that each siRNA specifically decreased secretion of the corresponding MMP without affecting secretion of the other MMP (Fig. 2B). The invasive capacity of the cells overexpressing mDia1-CA was investigated by silencing MMP expression. The invasion assay demonstrated that MMP-2-silenced MDA-MB-231 cells exhibited markedly lower mDial-CA overexpression-induced invasive capacity than MMP-9-silenced cells (Fig. 2C). These findings suggest that mDial is likely involved in the regulation of MMP-2 secretion to induce invasion of breast cancer cells. 
mDial promotes cell invasion via regulation of $M M P-2$ activity. To investigate whether MMP-2 secretion induced by mDia1-CA is sufficient to promote invasion of breast cancer cells, the effect of mDia1-CA expression on the activation of MMP and invasion of non-invasive breast cancer MCF-7 cells, which have lower mDia1 expression than MDA-MB-231 cells, was investigated (Fig. 3A). Gelatin zymography using conditioned media prepared from the mock- and mDia1-CA-transfected cells demonstrated that MCF-7 cells secrete MMP-2 and MMP-9 and that forced expression of mDia1-CA significantly induced MMP-2 activity, whereas MMP-9 activity was not markedly affected ( $\mathrm{P}<0.001$; Fig. $3 \mathrm{~B})$. The invasion assay indicated that control MCF-7 cells show little invasion into the membrane, whereas overexpression of mDia1-CA in MCF-7 cells resulted in significantly higher cell invasion than that of mock-transfected cells $(\mathrm{P}<0.001$; Fig. 3C). These results indicated that mDia1 is responsible for the invasive phenotype of breast cancer cells and MMP-2 is likely to mediate the invasion induced by mDial.

As mDial regulates actin filaments, previous studies have suggested that it is key in cell invasion via the actin assembly $(18,20,21)$. It has been reported that an actin-binding protein, cortactin, modulates the secretion and membrane expression of invadopodium-associated MMPs, including MMP-2 and MMP-9 in various cell types, such as vascular smooth muscle cells, head and neck squamous cell carcinomas, and breast cancer cells (22). As knockdown of mDia1 expression also reduces cortactin expression in malignant glioma cells (23), it is possible that mDia1 modulates MMP-2 and MMP-9 secretion via regulation of cortactin expression. In addition, results from the present study demonstrate that expression of MMP-2 is associated with breast cancer cell invasion induced by mDia1-CA (Fig. 2C). Although mDia1 and cortactin are important proteins for invasion in various cells, the detailed mechanism underlying the regulation of MMP secretion by $\mathrm{mDia}$ and cortactin requires further elucidation; this regulation may be cell type- and context-dependent.

MMP-2 and MMP-9 are highly linked to invasion of cancer cells, including breast cancer cells; however, the relative importance of MMPs for breast cancer cell invasion remains controversial (24-26). Although such effects may depend on experimental conditions, results from the present study demonstrated that increased invasion via mDia1-CA overexpression is specifically inhibited by knocking down MMP-2, while the effect of MMP-9 knockdown was not marked (Fig. 2C). These results suggest that MMP-2 and MMP-9 were involved in breast cancer cell activity; however, MMP-2 may be a major proteinase regulator of breast cancer cell invasion induced by mDia1.

In conclusion, the mDial protein expression level is elevated in a number of types of cancer, including kidney, prostate, and breast cancer. Overexpression of mDia1-CA promoted invasion of MDA-MB-231 cells, which was reduced by the addition of an MMP inhibitor. In addition, invasion induced by overexpression of mDia1-CA was significantly decreased by silencing MMP-2. Furthermore, overexpression of mDia1-CA induced invasion of MCF-7 cells due to increased MMP-2 activity. Results from the present study suggested that mDial is an important regulator of breast cancer cell invasion and that its effects may be mediated by MMP-2 activity.

\section{Acknowledgements}

The present study was supported by the National Research Foundation of Korea grant funded by the Korean government (grant no. NRF-2014R1A2A1A11050606).

\section{References}

1. Jemal A, Bray F, Center MM, Ferlay J, Ward E and Forman D: Global cancer statistics. CA Cancer J Clin 61: 69-90, 2011.

2. Yilmaz M and Christofori G: EMT, the cytoskeleton and cancer cell invasion. Cancer Metastasis Rev 28: 15-33, 2009.

3. Wang Y, Wen J and Zhang W: MIIP, a cytoskeleton regulator that blocks cell migration and invasion, delays mitosis and suppresses tumorogenesis. Curr Protein Pept Sci 12: 68-73, 2011.

4. Bartolini F and Gundersen GG: Formins and microtubules. Biochim Biophys Acta 1803: 164-173, 2010.

5. Wallar BJ and Alberts AS: The formins: Active scaffolds that remodel the cytoskeleton. Trends Cell Biol 13: 435-446, 2003.

6. Gaillard J, Ramabhadran V, Neumanne E, Gurel P, Blanchoin L, Vantard $M$ and Higgs HN: Differential interactions of the formins INF2, mDial and mDia2 with microtubules. Mol Biol Cell 22: 4575-4587, 2011.

7. Li F and Higgs HN: The mouse Formin mDial is a potent actin nucleation factor regulated by autoinhibition. Curr Biol 13: 1335-1340, 2003.

8. Shi Y, Zhang J, Mullin M, Dong B, Alberts AS and Siminovitch KA: The mDial formin is required for neutrophil polarization, migration and activation of the LARG/RhoA/ROCK signaling axis during chemotaxis. J Immunol 182: 3837-3845, 2009.

9. Magdalena J, Millard TH and Machesky LM: Microtubule involvement in NIH 3T3 Golgi and MTOC polarity establishment. J Cell Sci 116: 743-756, 2003.

10. Fernandez-Borja M, Janssen L, Verwoerd D, Hordijk P and Neefjes J: RhoB regulates endosome transport by promoting actin assembly on endosomal membranes through Dia1. J Cell Sci 118: 2661-2670, 2005.

11. Yamana N, Arakawa Y, Nishino T, Kurokawa K, Tanji M, Itoh RE, Monypenny J, Ishizaki T, Bito H, Nozaki K, et al: The Rho-mDial pathway regulates cell polarity and focal adhesion turnover in migrating cells through mobilizing Apc and c-Src. Mol Cell Biol 26: 6844-6858, 2006.

12. Stamenkovic I: Extracellular matrix remodelling: The role of matrix metalloproteinases. J Pathol 200: 448-464, 2003.

13. Stamenkovic I: Matrix metalloproteinases in tumor invasion and metastasis. Semin Cancer Biol 10: 415-433, 2000.

14. Schnaeker EM, Ossig R, Ludwig T, Dreier R, Oberleithner H, Wilhelmi M and Schneider SW: Microtubule-dependent matrix metalloproteinase-2/matrix metalloproteinase-9 exocytosis: Prerequisite in human melanoma cell invasion. Cancer Res 64: 8924-8931, 2004.

15. Egeblad M and Werb Z: New functions for the matrix metalloproteinases in cancer progression. Nature Rev 2: 161-174, 2002.

16. Sullu Y, Demirag GG, Yildirim A, Karagoz F and Kandemir B: Matrix metalloproteinase-2 (MMP-2) and MMP-9 expression in invasive ductal carcinoma of the breast. Pathol Res Pract 207: 747-753, 2011.

17. Ishikawa S, Takenaka K, Yanagihara K, Miyahara R, Kawano Y, Otake Y, Hasegawa S, Wada H and Tanaka F: Matrix metalloproteinase-2 status in stromal fibroblasts, not in tumor cells, is a significant prognostic factor in non-small-cell lung cancer. Clin Cancer Res 10: 6579-6585, 2004

18. Kitzing TM, Sahadevan AS, Brandt DT, Knieling H, Hannemann S, Fackler OT, Grosshans J and Grosse R: Positive feedback between Dia1, LARG and RhoA regulates cell morphology and invasion. Genes Dev 21: 1478-1483, 2007.

19. Kessenbrock K, Plaks V and Werb Z: Matrix metalloproteinases: Regulators of the tumor microenvironment. Cell 141: 52-67, 2010.

20. Lizárraga F, Poincloux R, Romao M, Montagnac G, Le Dez G, Bonne I, Rigaill G, Raposo G and Chavrier P: Diaphanous-related formins are required for invadopodia formation and invasion of breast tumor cells. Cancer Res 69: 2792-2800, 2009. 
21. Tanji M, Ishizaki T, Ebrahimi S, Tsuboguchi Y, Sukezane T, Akagi T, Frame MC, Hashimoto N, Miyamoto S and Narumiya S: mDial targets $\mathrm{v}-\mathrm{Src}$ to the cell periphery and facilitates cell transformation, tumorigenesis and invasion. Mol Cell Biol 30 4604-4615, 2010.

22. Clark ES, Whigham AS, Yarbrough WG and Weaver AM: Cortactin is an essential regulator of matrix metalloproteinase secretion and extracellular matrix degradation in invadopodia. Cancer Res 67: 4227-4235, 2007.

23. Li Z, Xu Y, Zhang C, Liu X, Jiang L and Chen F: Mammalian diaphanous-related formin 1 is required for motility and invadopodia formation in human U87 glioblastoma cells. Int J Mol Med 33: 383-391, 2013.
24. Baum O, Hlushchuk R, Forster A, Greiner R, Clézardin P, Zhao Y, Djonov V and Gruber G: Increased invasive potential and up-regulation of MMP-2 in MDA-MB-231 breast cancer cells expressing the beta3 integrin subunit. Int $\mathrm{J}$ Oncol 30: 325-332, 2007 .

25. Wang S, Liu Q, Zhang Y, Liu K, Yu P, Liu K, Luan J, Duan H, Lu Z, Wang F, et al: Suppression of growth, migration and invasion of highly-metastatic human breast cancer cells by berbamine and its molecular mechanisms of action. Mol Cancer 8: 81, 2009.

26. Lin CW, Shen SC, Hou WC, Yang LY and Chen YC: Heme oxygenase-1 inhibits breast cancer invasion via suppressing the expression of matrix metalloproteinase-9. Mol Cancer Ther 7: 1195-1206, 2008 